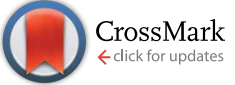

Cite this: RSC Adv., 2017, 7, 15372

\title{
Inkjet printing of nanocellulose-silver ink onto nanocellulose coated cardboard
}

\author{
Fanny Hoeng, ${ }^{\text {ab }}$ Julien Bras, ${ }^{\text {ac }}$ Erwan Gicquel, ${ }^{a}$ Guillaume Krosnicki ${ }^{\text {b }}$ \\ and Aurore Denneulin*a
}

Cellulose nanocrystals (CNC) have recently been used in the field of flexible electronics. The present work proposes to evaluate the contribution of $\mathrm{CNC}$ in the inkjet printing of silver conductive tracks. The potential of $\mathrm{CNC}$ to enhance both ink and substrate properties for printed electronics application was evaluated. First, CNC potential to be used as a scaffold for silver nanoparticles production in a compatible silver inkjet ink was evaluated. It has been found that the CNC dispersing properties, combined to their nanometrical rod-shape, allowed producing conductive suspensions at very low silver content comparing to classical silver ink. Inkjet printing of the CNC-silver suspension was then investigated on several substrates. Among these substrates, CNC coated cardboard has been studied to propose a new alternative for the printing of conductive tracks onto a porous substrate like cardboard. It appears that the CNC coating significantly limits the ink diffusion into the porous substrate by modification of the ink drop absorption kinetics, leading to the printing of well-defined conductive patterns. By using CNC in both ink formulation and substrate pre-treatment, the produced conductive patterns are nearly only composed of cellulosic material, offering new prospects in the development sustainable electronics.

Received 23rd September 2016
Accepted 3rd February 2017

DOI: $10.1039 / \mathrm{c} 6 \mathrm{ra} 23667 \mathrm{~g}$

rsc.li/rsc-advances
Inkjet printing process, in which a liquid ink is ejected through micrometric nozzles toward a substrate to produce patterned layers, is currently among the most used processes in printed electronics. Indeed, this non-contact and additive process allows the deposition of thin layers $(0.2$ to $2 \mu \mathrm{m})$ with a high resolution (up to line width of $40 \mu \mathrm{m}$ ). However, the particles size limitation to avoid nozzles clogging (particles diameter $<1 \mu \mathrm{m}$ ) and the specific ink properties requiring to allow droplet ejection (viscosity, surface tension specific range, colloidal stability) constitute the major limitations of this process. Among the different existing conductive inks used with the inkjet printing process, metallic nanoparticles inks are especially promising for designing electrically conductive connections. Indeed, given the high bulk conductivity of silver associated with its environmental resistance to oxidation, silver nanoparticles inks are today the most frequently used. However, the achievement of a high colloidal stability with such metallic particles appears still complex. Additives and solvents are generally needed for avoiding silver nanoparticles aggregates, which are not always tolerated within the inkjet printing process. ${ }^{10}$ In addition, when dealing with these metallic nanoparticles inks, a sintering post-treatment (comprise between 150 and $200{ }^{\circ} \mathrm{C}$ ) is necessary to ensure a metallic continuum for the electrical conduction, unlike larger silver particles which can be used in other printing processes like flexography or screen printing. The printed substrate must then be able to withstand the high level of temperature involved in this step without any degradation or deformations, which limits considerably the substrate choice.
${ }^{a}$ Univ. Grenoble Alpes, LGP2, F-38000 Grenoble, France.E-mail: aurore.denneulin@ grenoble-inp.fr

${ }^{b}$ Poly-Ink, 27, Boulevard Louise Michel, 92230 Gennevilliers, France

${ }^{c}$ Institut Universitaire de France (IUF), 75005 Paris, France 
Recently, nano-scaled particles coming from cellulose known under the name of nanocellulose have emerged in the field of printed electronics as detailed in a recent review. ${ }^{\mathbf{1 1}}$ Two type of nanocellulose currently exist: the cellulose nanofibrils (CNF) and the cellulose nanocrystals (CNC). CNC are small crystalline parts of cellulose fibers. An acid hydrolysis of the cellulosic fibers allows the isolation of those rigid rod like particles possessing dimensions within the nanometrical scale with a length ranging from 100 to $500 \mathrm{~nm}$ and a diameter ranging between 5 to $30 \mathrm{~nm}^{12}$ These renewable and biodegradable nanoparticles possess specific properties, such as high surface area and ability to form self-standing films. ${ }^{13}$ Their effectiveness in printed electronics was already demonstrated for providing self-standing smooth and/or transparent biodegradable substrates ${ }^{\mathbf{1 4 , 1 5}}$ answering the printed electronics requirements (i.e. a substrate presenting a smooth and closed surface with a relatively high dimensional stability over temperature). Their use as template to produce nano-structured metallic particles ${ }^{16-18}$ or as dispersing agent ${ }^{19,20}$ to stabilize metallic particles have also been recently studied. These hybrid systems were mostly implemented to produce antibacterial ${ }^{\mathbf{2 1 , 2 2}}$ or conductive ${ }^{17}$ composites but very few were evaluated for producing a conductive suspension dedicated to printed electronics, and especially to inkjet process. Indeed, several studies have printed conductive tracks on nanocellulosic substrate ${ }^{23-25}$ but no nanocellulose-based conductive inks have been proposed. Only one paper from Koga et al. emphasized the dispersing properties of nanocellulose to provide a conductive inkjet printable suspension of cellulose nanofibrils/carbon nanotubes. ${ }^{26}$

The present work proposes to evaluate the benefits of using $\mathrm{CNC}$ as a bio-based solution for the printing of conductive tracks: (i) first, the dispersing and stabilizing abilities of CNC were used to formulate conductive aqueous silver suspensions. These new developments are based on previous works, performed by our team, dealing with the synthesis of CNC-silver nano-structured particles. ${ }^{27}$ The suitability of CNC-silver hybrid suspension as conductive inkjet ink was evaluated. To fulfill all the inkjet printing requirements, investigations on suspension physico-chemical properties as well as a fine-tuning of the formulation were performed. Up to our knowledge, this is the first time that such study is performed on a CNC-silver suspension. Some works already reported the use of CNC in

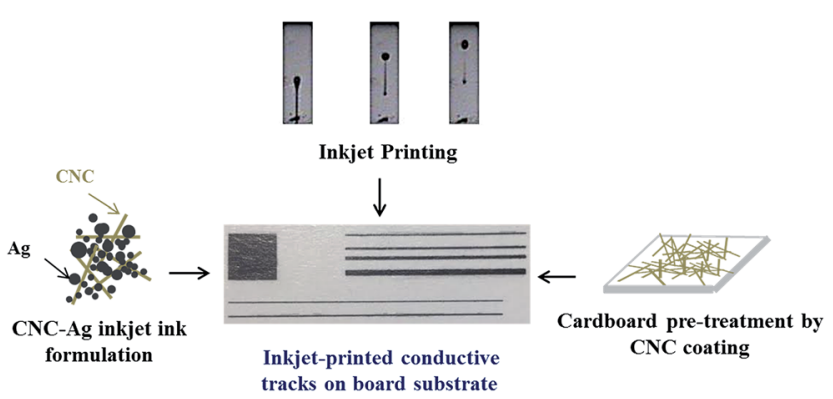

Fig. 1 Schematic representation of the CNC-silver inkjet printing on $\mathrm{CNC}$ coated substrate. inkjet printing but for biomedical application ${ }^{28}$ or tuning substrates $^{29}$ and inks ${ }^{30}$ properties but none of them have produced conductive suspension comprising CNC. (ii) Secondly, the ability of using a CNC coating as an easy alternative to provide suitable cellulosic porous substrates for printed electronics was evaluated and compared to other polymer films or printed electronics papers classically used. The printing quality of the CNC-silver ink was evaluated on different substrates including the CNC pre-treated substrate for achieving the best printability and optimal electrical performances. This work proposes for the first time to exploit CNC properties for printed electronics applications in both conductive inkjet ink and substrate coating as illustrated in Fig. 1.

\section{Experimental procedures}

\subsection{Materials}

Polyethylene terephthalate sheets (Lyreco, ref - 182.406, $100 \mu \mathrm{m}$ thickness), Powercoat® paper called PrintedElec paper (Arjowiggins, special printed electronic grade, $230 \mu \mathrm{m}$ thickness) and a cardboard were used as printing substrate. 2,2,6,6-Tetramethylpiperidine 1-oxyl (TEMPO), sodium hypochlorite (12\% wt), silver nitrate $\left(\mathrm{AgNO}_{3}\right)$, hexadecylpyridinium chloride monohydrate $\left(\mathrm{C}_{21} \mathrm{H}_{38} \mathrm{ClN} \cdot \mathrm{H}_{2} \mathrm{O}\right)$, sodium borohydride $\left(\mathrm{NaBH}_{4}\right)$ and dioctyl sodium sulfosuccinate salt $\left(\mathrm{C}_{20} \mathrm{H}_{37} \mathrm{NaO}_{7} \mathrm{~S}\right)$ were purchased from Sigma-Aldrich and used as received. Sulfated cellulose nanocrystals were purchased from University of Maine as an hydrogel $(7.26 \% \mathrm{wt})$. Distilled water was used in all experiments.

\subsection{TEMPO oxidation of CNC}

TEMPO mediated oxidation of CNC was performed using a previously described procedure in the literature. ${ }^{31} 11 \mathrm{~g}$ of CNC were dispersed by an ultrasonic probe treatment ( $5 \mathrm{~min}, 20 \mathrm{kHz}$ ) in $730 \mathrm{~mL}$ of water. TEMPO (325 mg, $2.08 \mathrm{mM}$ ) and $\mathrm{NaBr}$ (3.564 mg, $34.61 \mathrm{mM}$ ) were dissolved in $250 \mathrm{~mL}$ of deionized water and slowly added to the CNC suspension. A $12 \%$ wt $\mathrm{NaClO}$ suspension ( $66 \mathrm{~g}, 0.12 \mathrm{M}$ ) was then added dropwise to the suspension to start the oxidation. $\mathrm{pH}$ of the suspension was maintained around 10-10.5 by addition of $0.5 \mathrm{M} \mathrm{NaOH}$ solution while stirring the suspension for $3 \mathrm{~h}$. Reaction was then quenched by the addition of ethanol $(40 \mathrm{~mL})$ and the resulting CNC were washed with hydrochloric acid $0.5 \mathrm{M}$ at least three times by centrifugation (10000 rpm, $30 \mathrm{~min}$ ). After three centrifugations, the CNC were re-dispersed using the minimum of distilled water needed to recover all the CNC and put in dialysis against distilled water until a neutral $\mathrm{pH}$ was obtained. TEMPO CNC were stored in the fridge at constant neutral $\mathrm{pH}$ to allow the CNC to be in their carboxylate form.

\subsection{Conductive ink formulation}

Silver conductive ink suspension was synthetized following our previously described procedure. ${ }^{32}$ Produced oxidized CNC were used as both capping and stabilizing agent in the synthesis of silver nanoparticles: $200 \mathrm{~mL}$ of a $0.5 \% \mathrm{wt}$ CNC suspension at neutral $\mathrm{pH}$ was mixed with $200 \mathrm{~mL}$ of a $0.5 \mathrm{mmol} \mathrm{L}^{-1}$ 
hexadecylpyridinium chloride solution during 5 minutes. 200 $\mathrm{mL}$ of $\mathrm{AgNO}_{3}\left(10 \mathrm{mmol} \mathrm{L}^{-1}\right)$ was then added to the suspension and stirred during 5 additional minutes. Finally, $200 \mathrm{~mL}$ of a reducing agent solution $\left(\mathrm{NaBH}_{4} / 30 \mathrm{mmol} \mathrm{L}{ }^{-1}\right)$ was added and the suspension was stirred for another 5 minutes. The $\mathrm{NaBH}_{4}$ solution was freshly prepared prior each experiment. The resulting suspension was then centrifuged three times (10 $000 \mathrm{rpm}, 20$ minutes). The final sediment was re-dispersed in needed amount of deionized water by mechanical agitation followed by an ultra-sonic probe treatment $(20 \mathrm{kHz}, 3 \times 15 \mathrm{~min})$ to obtain a stable suspension.

\subsection{Inkjet printing of CNC-silver system}

A laboratory piezoelectric inkjet printing machine (Fujifilm Dimatix DMP 2831 with 10 pL nominal drop size cartridge) was used to print the developed conductive suspension. This equipment allows the loading of disposable piezoelectric inkjet print heads including 16 nozzles of $21.5 \mu \mathrm{m}$ (square shape) and distanced at $254 \mu \mathrm{m}$ from each other. Prior to each experiment the inkjet print heads were degassed using a syringe and the purge system embedded on the printer. Printing parameters such as drop spacing, nozzle temperature and print height were set according to Table 1. Substrate temperature was set to $30^{\circ} \mathrm{C}$ during the printing.

Ejection parameters (voltage and waveform design) were optimized in preliminary adjustments. Cleaning cycles were performed every 50 printed bands to avoid temporary nozzle clogging and substrate contamination. Patterns including lines with variable widths $(0.1$ to $0.5 \mathrm{~mm})$ and solid areas of $10 \times 10$ $\mathrm{mm}$ square were printed to evaluate the print quality and the electrical performances. After the printing, samples were allowed to dry at ambient air overnight. For conductivity measurement, annealing post-treatment $\left(180^{\circ} \mathrm{C}\right.$ to $\left.30 \mathrm{~min}\right)$ was performed in an oven to allow the sintering of silver nanoparticles. In addition, a drop watcher camera coupled with a drop volume measurement module was implemented to determine characteristics such as drop shape, drop velocity, and drop volume.

\subsection{CNC coated substrate preparation}

To meet the printed electronics requirements regarding substrate, a $10 \%$ wt CNC suspension was coated on the cardboard samples with a bar coater (Kcoater 301, Erichsen). To obtain a regular and thin film, a non wire-wound Mayer bar was used with a coating speed of $5.4 \mathrm{~cm} \mathrm{~s}^{-1}$. This configuration allows producing coating thickness of approximately $0.8 \mu \mathrm{m}$ (SEM measurements). The coated cardboards were then dried under ambient air overnight.

Table 1 Inkjet printing parameters summary

\begin{tabular}{lll}
\hline Drop spacing $(\mu \mathrm{m})$ & Print height $(\mathrm{mm})$ & Ink temperature $\left({ }^{\circ} \mathrm{C}\right)$ \\
\hline
\end{tabular}

15

1

28

\subsection{Suspension characterization}

All the errors bars presented in the measurement represent the standard deviation of the measurement series.

Conductimetric titration. Charge density of oxidized CNC was measured conductimetric titration. About $15 \mathrm{mg}$ of CNC were put into $50 \mathrm{~mL}$ of distilled water and treated by ultrasonic bath for 5 minutes. $\mathrm{pH}$ of the suspension is adjusted to 2 by adding $\mathrm{HCl} 0.1 \mathrm{M}$ to allow carboxylate groups to be in their protonated form. The suspension is then titrated against $0.1 \mathrm{~mL}$ increment of $\mathrm{NaOH} 0.01 \mathrm{M}$.

Surface tension. Surface tension of inks was measured using the Du Noüy ring method on a tensiometer KRÜSS K10ST using a $2 \mathrm{~cm}$ platine ring. Measurements were performed at ambient air and were repeated 10 times for each suspension to achieve a representative value.

Rheological measurement. The rheological behavior of the developed suspensions was studied using a rotational rheometer (MCR02, Antonn Paar) with a cone-plate geometry (diameter $=50 \mathrm{~mm}$, truncate $=55 \mu \mathrm{m}$, angle $=1.013^{\circ}$ ) and a set gap of $0.112 \mathrm{~mm}$. A logarithmic ramp with shear rate varying from 0.1 to $1000 \mathrm{~s}^{-1}$ was performed to monitor the ink rheological behavior and viscosity. Viscosity value at $1000 \mathrm{~s}^{-1}$ was kept to be the most representative of shear rate induced during the inkjet printing process. Indeed, shear rates generated within an inkjet print head can be extremely high, i.e. up to $10^{5}$ to $10^{6} \mathrm{~s}^{-1}$ (combination of microscale ink channels with high volume flow rates). Three measurements were performed to check the reproducibility of the measure.

Thermogravimetric analysis. Suspension solid content and silver content were determined using thermogravimetric analysis (STA6000@, Perkin-Elmer) with a temperature ramp of $10{ }^{\circ} \mathrm{C}$ $\mathrm{min}^{-1}$ under air. Samples were dried for one night at $50{ }^{\circ} \mathrm{C}$ prior to the analysis for eliminating the water and precisely determine the suspension composition. 3 duplicates were performed and average data are presented.

\subsection{Substrate and printed patterns characterization}

Substrate selection and characterization. Each tested substrate has been characterized in terms of thickness, roughness and surface energy. Basis weight and thickness have been measured according to the ISO 536:1995 and ISO 534:2005 standards. Surface energy measurements were carried out with the contact angle method on an OCA dataphysics device, in accordance with the Owens-Wendt model using four different liquids (water, hexadecane, ethylene glycol and diiodomethane). Ink drop volume $(5 \mu \mathrm{L})$ evolution on selected substrate was also evaluated using the same apparatus.

Microscopy analysis. Morphology of the conductive deposit was firstly observed by optical microscopy to characterize print quality. Images were taken along the $200 \mu \mathrm{m}$ width printed line and 10 images per samples were recorded. Observation at a nano-scale were also performed using a Zeiss Ultra 55@ field emission gun-scanning transmission electron microscope (SEMFEG). Secondary electrons images were obtained after a metallization step of the sample (Au/Pd layer $-1 \mathrm{~nm}$ ) at an accelerating voltage (EHT) of $5 \mathrm{kV}$ and a working distance of $6.3 \mathrm{~mm}$. 
Observations at magnifications of 20000,50000 and 100000 were performed and at least 10 images per sample were recorded. A focus has been done to characterize the nanoparticles network (organization, distribution) within the printed layers. Thickness of the printed conductive films was also evaluated by analysing the cross sections at different locations using an EHT of $3 \mathrm{kV}$ at magnifications of 8000 and 15000 .

AFM. Atomic force microscopy was used to image the CNC morphology and the printed CNC-silver network on a Dimension Icon Bruker ${ }^{\circledR}$. Scans of 3.3 and $10 \mu \mathrm{m}^{2}$ were obtained using tapping mode and silicon coated tips (O-TESPA). At least 10 images per samples were recorded and the most representative images were kept for discussion.

Surface roughness. Surface roughness was measured using AFM images on $20 \times 20 \mu^{2}$ scans. 15 measurements were realized and the average data is presented for the results.

Electrical properties. Electrical properties were characterized by measuring the sheet resistance of the printed films. Measurements were taken using a Jandel Universal four-probes system, connected to a Jandel RM3 current generator providing a current range from $10 \times 10^{-9}$ to $99 \times 10^{-3} \mathrm{~A}$. In this configuration, the two external probes impose a known current intensity and the two internal probes measure the corresponding voltage. The expression of the sheet resistance in this particular configuration is defined below:

$$
R_{\text {sheet }}=K \times \frac{U}{I}\left[\Omega \square^{-1}\right]
$$

with $K$ a constant depending on the geometry, $U$ the measured voltage $(\mathrm{V})$ and $I$ the set intensity (A).

To limit edge effects, measurements were performed on solid area patterns of $10 \times 10 \mathrm{~mm}$ square. 10 measurements have been performed and the averaged value is presented.

\section{Results \& discussions}

\subsection{Conductive ink preparation and selection}

TEMPO oxidized CNC are characterized by the presence of surface carboxylate groups. ${ }^{31}$ In this work, TEMPO CNC were used to act both as capping and stabilizing agent for silver nanoparticles synthesis. ${ }^{32}$ Indeed, in previous studies, we emphasize the importance of using oxidized CNC with a high surface charge density to enhance the silver synthesis on their surface and obtain a stable suspension. ${ }^{32}$ Moreover, the use of a cationic surfactant during the synthesis as well as the reactants ratios were identified as key factors to achieve stable silver aqueous suspensions with the highest silver loading. ${ }^{27}$ Considering the previous results, oxidized CNC with a high charge density of carboxylate moieties were used. The highly charged CNC ensure the suspension stability by the presence of repulsive interactions while allowing a better control of the silver synthesis. ${ }^{32}$ The charge density of our CNC was determined to be $1300 \pm 51 \mu \mathrm{mol} \mathrm{g}^{-1}$ using conductimetric titration. ${ }^{31}$ The CNC dimensions were measured to be $200 \pm 19 \mathrm{~nm}$ in length and $20 \pm 2 \mathrm{~nm}$ in diameter using AFM. These CNC nanometrical dimensions are essential given the inkjet particle size limitation ( $<1 \mu \mathrm{m}$ to avoid nozzles clogging).

Synthesis of the silver nanoparticles on CNC was done according to a previously presented protocol. ${ }^{27}$ An optimal CNC to silver ratio was used to control the silver nanoparticles synthesis based on preliminary experiments (not presented here). With the aim of achieving stable conductive suspension, impact of silver amount in the suspension was first evaluated on the suspension stability and conductivity. Suspensions electrical properties were estimated by a drop casting technique. ${ }^{33} \mathrm{~A}$ $5 \mu \mathrm{L}$ drop was deposited on a printed electronic paper followed by a sintering at $180{ }^{\circ} \mathrm{C}$ during $30 \mathrm{~min}$ (10 measurements for each suspension). This preliminary approach was performed in order to select conductive suspensions. Table 2 summarizes the characteristics of each prepared suspension.

Surprisingly, the suspension starts to be conductive at a silver content around 3\% wt Considering that the percolation rate of conventional silver nanoparticle suspensions is around $20 \% \mathrm{wt}$, we believe that, in our case, a percolated network was achieved at a lower silver content due to changes in the network organization happening with the CNC. When increasing the silver amount above $3 \mathrm{wt} \%$, the suspension became unstable with visible silver particles sedimentation occurring after only 1 day. This loss in suspension stability can be explained by changes in electrostatic interactions between the CNC-silver particles. As the CNC-silver content in the suspension is increased, the distance between each CNC-silver particle is decreased due to a decrease in available spatial volume. This change in inter-particles distance leads to changes in their interactions. Starting a given concentration of silver, the attractive silver-silver interactions then became dominant over the CNC anionic repulsive forces, therefore inducing the particles aggregation and sedimentation.

The 3\% wt silver suspension was thus selected for evaluating the suitability of the suspension to be processed by inkjet printing. The synthetized silver particles dimensions within this suspension was estimated to $64 \pm 16 \mathrm{~nm}$, which should be in accordance, as for CNC particles, with the size limitation imposed by the inkjet printing process.

Table 2 Properties the different CNC-silver suspensions

\begin{tabular}{lllll}
\hline & CNC content $(\% \mathrm{wt})$ & Silver content $(\% \mathrm{wt})$ & $5 \mu \mathrm{L}$ drop resistance $(\Omega)$ & Sedimentation time $(\mathrm{days})$ \\
\hline CNC-silver1 & $0.4 \pm 0.4$ & $1.0 \pm 0.1$ & Not conductive & Stable \\
CNC-silver2 & $0.9 \pm 0.3$ & $2.1 \pm 0.1$ & Not conductive & Stable \\
CNC-silver3 & $1.2 \pm 0.4$ & $2.9 \pm 0.2$ & $0.31 \pm 0.01$ & Stable \\
CNC-silver4 & $2.1 \pm 0.1$ & $4.2 \pm 0.2$ & $0.12 \pm 0.02$ & 1 day (unstable)
\end{tabular}




\subsection{Optimization of CNC-silver suspension for inkjet process}

Physico-chemical properties of the selected CNC-silver hybrid formulation, namely rheological behavior and surface tension, were first evaluated to determine the suitability of the suspension regarding inkjet printing process. The rheological behavior of the CNC-silver suspension (not presented here) shows a shear thinning behavior against the whole range of shear rate. Besides the shear thinning behavior, the viscosity of the suspension at $1000 \mathrm{~s}^{-1}$ was estimated to $1.2 \mathrm{mPa}$, which is in accordance with the low-viscosity window targeted for the inkjet printing process.

The suitability of the suspension for inkjet printing was evaluated by the mean of a dimensionless number analysis. Indeed, in piezoelectric inkjet printing process, the ink droplet is ejected thanks to an electromechanically impulsion induced by a piezoelectric element. The droplet is jetted only if its kinetic energy overcomes interactions linked to surface energy and viscous forces. Both conditions are formulated through the dimensionless numbers Weber $\left(\mathrm{We}=\frac{\rho v^{2} d}{\gamma}=\frac{\text { inertial effects }}{\text { capillary effects }}\right)$ and Reynolds $\left(\operatorname{Re}=\frac{\rho v d}{\eta}=\frac{\text { inertial effects }}{\text { viscous effects }}\right)$, which must be superior to 1 for ensuing droplet ejection. We number describes the kinetic-surface energy conversion governed by the speed of the jet and the surface tension. Re number takes into account the viscosity effects. An approach combining these two dimensionless numbers for predicting a fluid inkjettability was firstly proposed by Fromm et al. and was refined later by Derby et $a l^{34,35}$ In these studies, the fluid properties are described by the $Z$ number, which is the inverse of Ohnesorge number $(\mathrm{Oh})$. Oh characterizes the propagation of the pressure wave and its attenuation by viscous dissipation. The $Z$ number is then defined as below in eqn (2).

$$
Z=\frac{1}{\mathrm{Oh}}=\frac{\mathrm{Re}}{\sqrt{\mathrm{We}}}=\frac{\sqrt{\rho \sigma d}}{\eta}
$$

where: $\rho=$ volumic mass $\left(\mathrm{kg} \mathrm{m}^{3}\right), v=\operatorname{droplet}$ speed $\left(\mathrm{m} \mathrm{min}^{-1}\right)$, $\sigma=$ surface tension of the ink ( $\mathrm{N} \mathrm{m}), d=$ nozzle diameter $(\mathrm{m}), \eta$ $=$ dynamic viscosity of the ink (Pa s).

Fromm et al. have predicted that a suspension can be ejected by inkjet nozzle only if $Z>2$. Following this work Derby and Reis specified that a stable droplet formation is obtained for $1 \leq Z \geq$ 10. Finally, Jang et al. redefined then the printable range as $4 \leq$ $Z \geq 14$ considering additional parameters like droplet generation and positional accuracy. ${ }^{36}$ Printing using a fluid with $Z<4$ will lead to a drop formation with undesired long-lived filament while inks with $Z>14$ would not be able to form a single droplet without undesired satellites drops.

Table 3 (first line) summarized the suspension properties and associated dimensionless number. Calculations were made considering a nozzle diameter of $21.5 \mu \mathrm{m}$ and a suspension density of $996 \mathrm{~kg} \mathrm{~m}^{3}$. As it can be seen, the $Z$ number calculated for the CNC-silver suspension $(Z=20)$ appears too high to allow a correct ejection of the ink. Considering the high surface tension of $50.1 \mathrm{mN} \mathrm{m}^{-1}$ of the ink, formulation adjustments were performed to lower this value by adding different amount of dioctyl sulfosuccinate (DSS) surfactant. Table 3 summarized the characteristics of the different suspension prepared with the associated evolution of the surface tension and $Z$ number. As expected, the surfactant addition leads to a decrease in surface tension and an acceptable value of $Z(Z=13.3)$ was reached for a minimum concentration in surfactant of $0.15 \%$ wt, which corresponds to a surface tension of $29.1 \mathrm{mN} \mathrm{m}^{-1}$. As surfactant molecules are known for being insulative and can thus affect the conductivity of our system, the lowest surfactant concentration required to meet inkjet requirements $(0.15 \% \mathrm{wt})$ was selected for the next results.

Inkjet printing of the selected suspension was then evaluated. No nozzles clogging and with a stable droplet formation have been observed during the printing. This printing stability is in good agreement with the previous theoretical expectations. It is worth mentioning that even after 1 month, no additional clogging was observed with the same print head, confirming the high stability of the ink.

Optimization of inkjet printing parameters was done to obtain the most spherical drops. The firing voltage was thus set to $30 \mathrm{~V}$ with an associated waveform presented in Fig. 2a.

Resulting drops in Fig. $2 b$ shows that single spherical droplets with a small tail are fired, in good agreement with the previously calculated $Z$ number of 13.3. The microscope image in Fig. 2c shows that the preliminary printed drops on printed electronics paper possess a spherical shape with no satellite drops around the printing. These results suggest that the selected suspension was indeed suitable to provide inkjet printed tracks.

Advantage of the suspension especially lies on the use of CNC shape for obtaining a conductive inkjet ink at low solid content due to the use of $\mathrm{CNC}$ and their percolation ability.

Classical silver inks are usually conductive with $20 \%$ wt of silver in the suspension. Thus, by using the CNC-silver

Table 3 Physico-chemical properties of CNC-silver suspension with different amount of surfactant and associated calculated dimensionless number

\begin{tabular}{|c|c|c|c|c|c|c|c|}
\hline CNC-silver & 0 & $1.2 \pm 0.3$ & $50.1 \pm 0.7$ & 15 & 43 & 133 & 20.4 \\
\hline DSS (0.15) & 0.15 & $1.9 \pm 0.4$ & $29.1 \pm 0.1$ & 14 & 71 & 101 & 13.3 \\
\hline DSS (0.2) & 0.2 & $1.9 \pm 0.3$ & $26.7 \pm 0.3$ & 14 & 80 & 112 & 12.5 \\
\hline
\end{tabular}


(a) Jetting Waveform

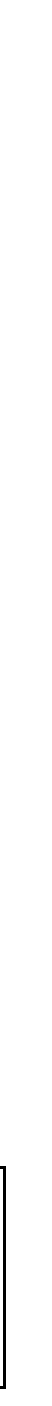

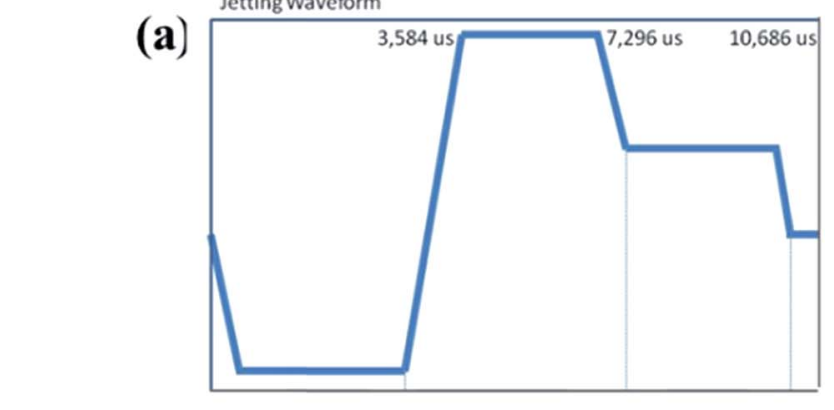

(b)

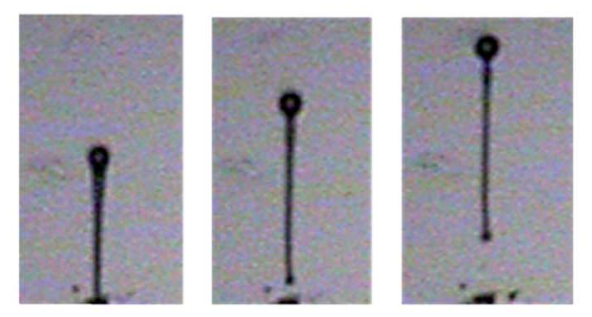

(c)

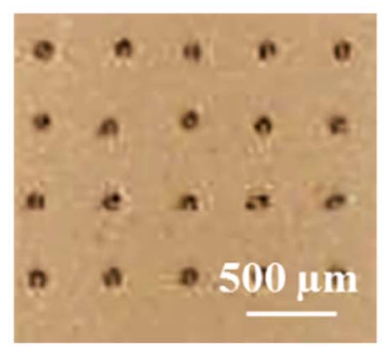

Fig. 2 (a) Inkjet waveform; (b) photograph of CNC-silver fired drops and (c) optical microscope image of inkjet printed droplets.

suspension, the cost in silver material is decreased with only $3 \%$ of silver used. Another advantage lie on the aqueous stability, which is very difficult to obtain with silver nanoparticles. Thank to the use of $\mathrm{CNC}$, the suspension can be stable in water.

\subsection{Influence of substrate properties on CNC-silver ink printability}

The optimized selected CNC-silver suspension was printed onto classical printed electronics substrates, namely PET plastic foil and printed electronic Powercoat ${ }^{\circledR}$ paper (PrintElec paper). A neat porous cardboard was also evaluated as a cellulosic reference substrate for conductive printing. To prepare the cardboard surface, a CNC coating was tried out to meet the printed electronic requirements. Indeed, it has been shown that nanocellulose films can provide green and renewable substrates suitable for the printing of conductive tracks. ${ }^{23,24}$ However, no work has evaluated the impact of a nanocellulose coating on classical cellulosic substrates. Thus, with the aim of (i) studying the influence of a CNC coating on the printability of our ink and (ii) evaluating the suitability of such coating for the printing of conductive tracks, a CNC suspension was coated on the porous
Table 4 Characteristic surface properties of the different tested substrate

\begin{tabular}{llr}
\hline Substrate & $\begin{array}{l}\text { Surface energy } \\
\left(\mathrm{mN} \mathrm{m}^{-1}\right)\end{array}$ & \multicolumn{1}{l}{$\begin{array}{l}R_{\mathrm{a}} \text { roughness } \\
(\mathrm{nm})\end{array}$} \\
\hline PET & $41 \pm 1$ & $5.3 \pm 0.5$ \\
Printed Elec paper & $32 \pm 4$ & $10.1 \pm 7.4$ \\
Board & $36 \pm 5$ & $66.2 \pm 8.1$ \\
Board + CNC & $49 \pm 4$ & $3.6 \pm 1.2$ \\
\hline
\end{tabular}

cardboard substrate. The obtained properties were compared to that of the neat paperboard substrate and the classical printed electronics substrates.

The different substrates properties, summarized in Table 4 , shows that the selected CNC-silver ink should theoretically have appropriate interactions with all substrates. Indeed, as the surface tension of the suspension is inferior to the surface energy of each substrate, a good wetting of the substrate by the ink should be observed. The printing quality achieved on each substrate was analyzed using optical microscope imaging. Observations were made on both individual printed drops and $200 \mu \mathrm{m}$ printed lines. Observations of printed lines in Fig. 3 show an inhomogeneous printing on PET and PrintElec paper with an unequal ink repartition along the line, which is also evidenced on the printed solid areas (not presented here).

A coffee ring effect is visible on both substrates with higher material amount on the edge of the printed patterns. This is also confirmed by the inset images in Fig. 3, showing the individual printed drop on the different substrates. The coffee ring effect is clearly evidenced with a material reorganization towards the edge of the pattern. The coffee ring effect is a wellknown studied printing defect (first described by Deegan et al., 1997), especially occurring with the inkjet printing process due to the low viscosity of the fluid. This phenomenon is induced during the droplet drying phase ${ }^{37}$ by the combination of two actions: (i) the solvent evaporation is more important at the edge of drops and (ii) the surface contact area between ink and substrate is pinned for the major part of the drying. A flow takes place to compensate the solvent removal by evaporation at the edge of droplet. The solvent, and subsequently the suspended particles, can then be totally or partially transferred to the contact line by this flow and generate a ring shape to the dried droplet. Additional unequal ink repartition is visible on both PET and powercoat ${ }^{\circledR}$ printed lines and might be due to a liquid film reorganization happening during the printing. This phenomenon was already described as bulging defect by Duineveld $^{38}$ and later Soltman ${ }^{39}$ who suggested that when the contact angle - due to additional fluid from printing - is higher to that of equilibrium contact angle, a liquid reorganization within the printed film happened despite a theoretical inksubstrate compatibility. ${ }^{38}$ Thus, we believe that our ink is leading to both of these defects on both PET and PE paper, especially due to the fact that it is water-based. Indeed, several studies demonstrated that water-based ink favors the coffee ring due to a non-uniform evaporation of the volatile water as compared to solvent based suspension..$^{40}$ This phenomenon is 


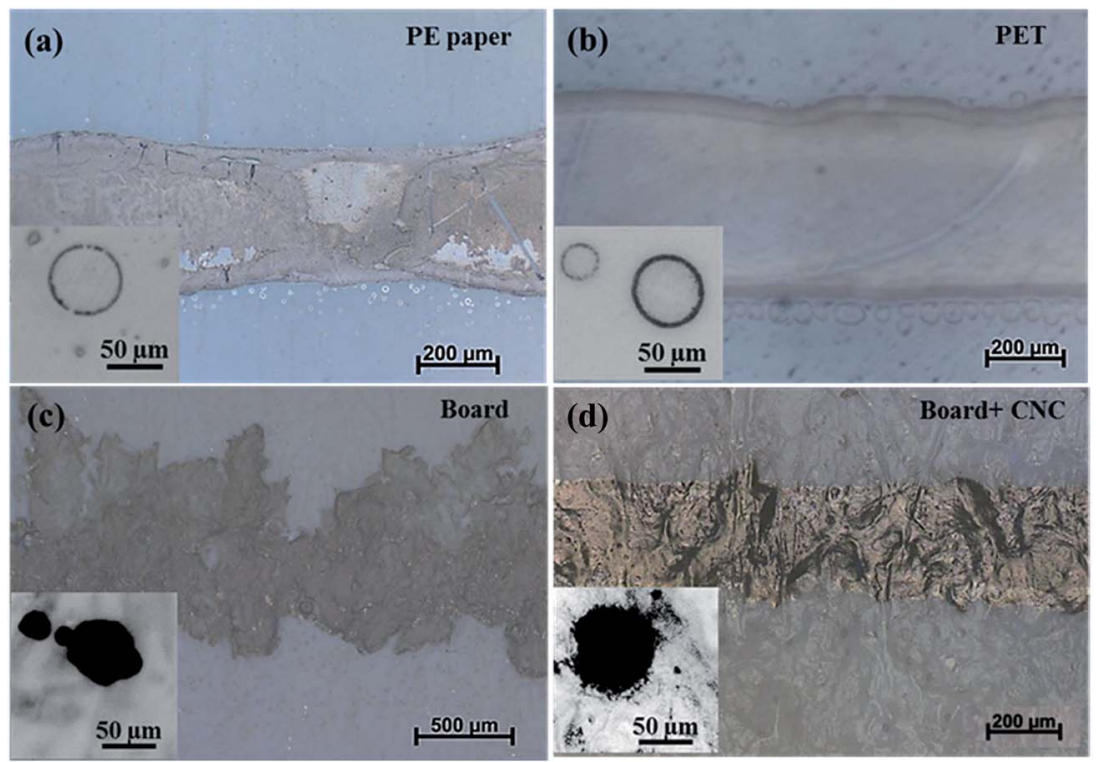

Fig. 3 Optical microscope images of a $200 \mu \mathrm{m}$ printed line of CNC-silver suspension on (a) printed electronic paper; (b) PET; (c) neat board and (d) CNC coated board substrate.

usually compensate by adding a less volatile co-solvent within the ink formulation. ${ }^{41}$ However, in our case, this solution has not been considered for developing a $100 \%$ aqueous based ink.

The printing on neat cardboard evidenced classical printing defects on porous substrate, with visible ink infiltration and spreading onto the substrate. The ink infiltration into the substrate leads to high line widening of $75 \%$. Interestingly, when printing on the CNC coated cardboard substrate a welldefined line was obtained with a smaller line widening of $24 \%$ as compared to neat board. These results are in accordance with a recent patent who described an increased printability for a CNC coated plastic substrate. ${ }^{29}$

Comparison of the printing on different substrates evidenced a better printing quality on the CNC coated cardboard. This higher printing quality obtained on the CNC coated substrate can originate from different parameters such as differences in substrate roughness, porosity (or capillary absorption) or surface energy. Previously introduced Table 4, comparing the roughness of the different substrates, shows that the roughness of the cardboard is divided by almost 20 when using a CNC coating. These results, demonstrating the effectiveness of a CNC coating to lower a substrate roughness, can explain the more homogeneous material deposition. However, the roughness of the coating remains higher to that of classical printed electronics substrates. The inset images of the printed drop on the CNC coating show more irregular shape than the spherical ones obtained on PET and PrintElec paper, probably due to this higher roughness. Consequently, considering the more regular shapes on PET and PrintElec, the roughness alone does not explain the better line definition obtained on the CNC coating.

Some studies have demonstrated the importance of ink penetration for the printing of conductive tracks on paper. ${ }^{42,43}$ In our case, we believe that the use of a CNC coating would modify the ink penetration inside the substrate. Indeed, the very dense and close nanometrical network formed by the CNC coating probably impacts the substrate liquid absorption surface properties, thus explaining the better print quality on the treated cardboard substrate. To confirm this hypothesis, drop volume time-dependent measurements were performed on both neat and CNC coated cardboard to evaluate the ink absorption kinetic. Considering that evaporation rate of inkjetprinted drops occurs fastly for water-based suspensions $(\sim 800$ ms), ${ }^{44}$ measurements were done on a short time period. Fig. 4 presents the drop volume evolution against time of the CNCsilver ink on neat cardboard and CNC coated board substrate.

When deposited on the CNC coated substrate, the volume of the drop shows an immediate decrease (see the inset of the figure) before reaching a stable value after only $\approx 0.2$ seconds. On the contrary, when deposited on neat cardboard the ink drop volume shows a slow decrease up to $30 \mathrm{~s}$, due to the

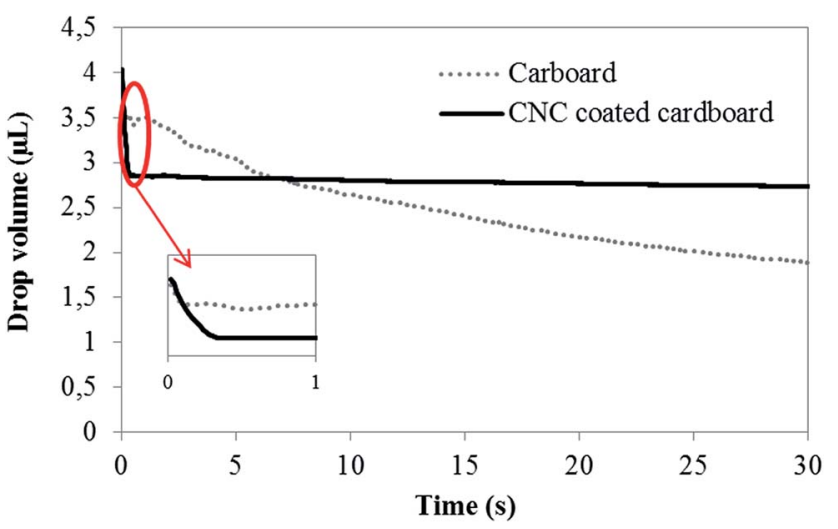

Fig. 4 Drop volume evolution of the CNC-silver ink on the CNC coated board and neat board using contact angle experiment. 
diffusion of the ink inside the substrate. This result, representative of a faster ink absorption by the CNC coated cardboard as compared to the long and continuous absorption of the neat cardboard, can explain the different print quality obtained on the two substrates. Indeed, absorption kinetics presented in Fig. 4 clearly evidenced that the CNC coating increase the ink absorption speed by the substrate, probably due to the high surface area of the nanoscale CNC compared to neat cellulose fiber of the cardboard. Over a period of $800 \mathrm{~ms}$, the average absorption speed for the CNC coating has been calculated to be $1.5 \mu \mathrm{L} \mathrm{s}^{-1}$ against only $0.5 \mu \mathrm{L} \mathrm{s}^{-1}$ for the neat cardboard. Consequently, when a printed drop reach the CNC coated substrate, absorption of the drop happens so fast that the printed drop would not have time to diffuse inside the substrate. It worth mentioning that drop volumes in inkjet printing are much lower $(\sim \mathrm{pL})$ than the one studied, suggesting that these effects happened even faster during the printing of the CNC-silver suspension.

Additionally, this faster absorption kinetic might also explain the absence of coffee ring on the CNC coating as compared to PET or PrinElec paper substrate. Indeed, volume of drops fired by inkjet can be considered to be around 2 to 70 pL. ${ }^{44,45}$ Using the previously calculated average speed, the maximal drop volume of $70 \mathrm{pL}$ would be absorbed in about 46 ms by the CNC coating whereas the evaporation time of an aqueous inkjet drop was estimated to be around $800 \mathrm{~ms} .{ }^{44}$ Thus, the flux at the origin of the coffee ring effect, which happens during the evaporation stage of the drop, would not have time to occur. This hypothesis, schematically illustrated in Fig. 5, also explains the higher printing quality and definition obtained on the CNC coated substrate.

The previous results evidenced that the best printing quality was obtained on the cardboard coated CNC substrate with a good line resolution and limited ink infiltration. This $100 \%$ cellulosic substrate can provide a green solution for the printing of conductive tracks with the advantage of possessing a higher thermal stability than plastic based substrate. As compared to a $100 \%$ nanocellulosic substrate, the coating solution provides

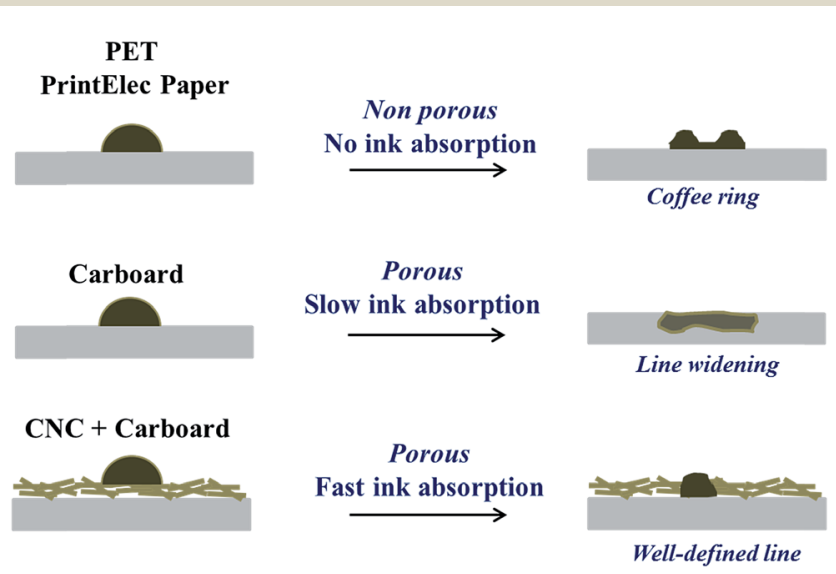

Fig. 5 Schematic illustrations of the mechanisms happening after drop impact on the different substrates. an easy way for tuning substrate properties using a low amount of nanocellulosic material.

\subsection{CNC-silver ink electrical properties}

According to the previous results, the best print quality was obtained on the CNC coated paperboard substrate. Electrical properties of the ink were then evaluated using this substrate. It is well known that silver nanoparticles network became conductive after an annealing treatment needed for forming a continuous silver network. Fig. 6 represents the evolution of the sheet resistance according to the temperature and associated network evolution.

As it can be seen, at intermediate annealing temperature (30 min to $180{ }^{\circ} \mathrm{C}$ ), the obtained sheet resistance of about $50 \Omega$ $\square^{-1}$ appears quite high as compared to obtained performances of classical silver nanoparticle inks reported in the literature. ${ }^{\mathbf{4 6}}$ However, considering the silver content of $3 \% \mathrm{wt}$ in the suspension, it is not surprising that conductivity is lower to that of classical silver inks containing at least $20 \% \mathrm{wt}$ of silver. ${ }^{47}$ In addition, the presence of insulative surfactant and nanocellulose around the silver particles visible in both AFM image of the network in Fig. 6 suggests that the CNC presence limits the network conductivity, leading to a degraded sheet resistance value. This was confirmed by the evolution of the sheet resistance happening when increasing the annealing temperature around the cellulose degradation temperature $\left(250{ }^{\circ} \mathrm{C}\right)$. The sheet resistance dramatically decreases from $50 \Omega \square^{-1}$ and to 1 $\Omega \square^{-1}$ with a clear loss of the visible CNC on AFM images. Thus, although CNC ensure the stability of the silver suspension, the insulative nature of CNC appears as a limitation to achieve high conductive patterns at low sintering temperature. However, it is important to highlight that despite the very low silver content loaded in the suspension, an interesting level of electrical conductivity was achieved with sufficient annealing treatment. This is probably due to the use of CNC, which allows a significant decrease of the silver particles percolation threshold. Indeed, the high aspect ratio of CNC acts as a scaffold for silver nanoparticles allowing a network organization, resulting in a decrease of the network percolation threshold. SEM images in Fig. 7 confirm this hypothesis with visible silver

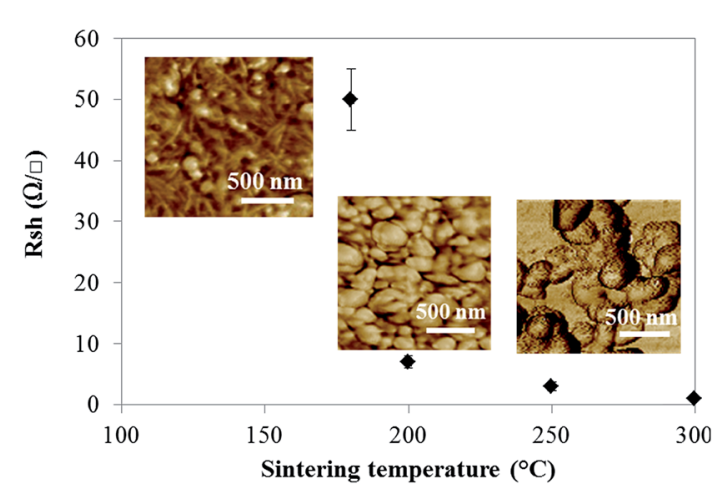

Fig. 6 Evolution of the sheet resistance against the sintering temperature and associated with AFM images of the network. 

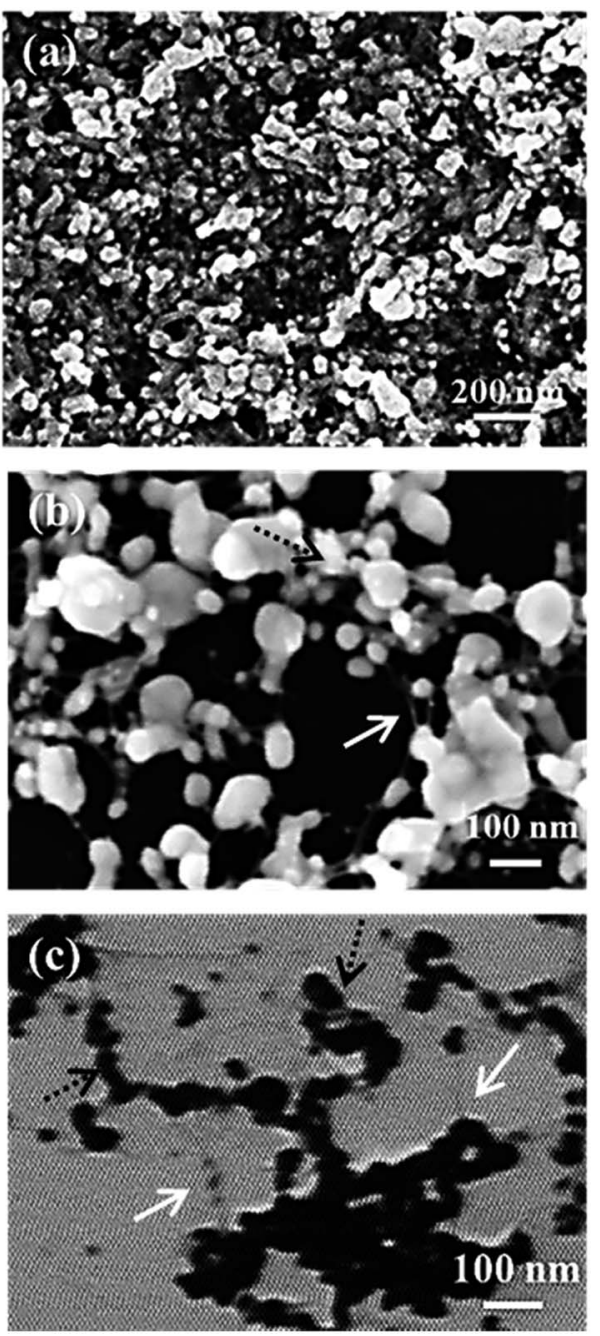

Fig. 7 SEM images of the $\mathrm{CNC}$-silver ink after sintering at $180^{\circ} \mathrm{C}$ to 30 min for (a) the dried CNC-silver printed film, (b) the diluted CNCsilver ink and (c) STEM image of the same diluted and sintered CNCsilver ink.

particles (black dotted arrows) onto the CNC (white arrows) and no free silver particles.

This decrease in the percolation threshold when using CNC has already been reported for conductive polymers composite films ${ }^{48,49}$ but, as far as we know, this is the first time that it has been reported for conductive silver particles. Considering these results, we believe that both the insulative cellulose and the silver solid content in the suspension limits the suspension conductivity. However, the use of $\mathrm{CNC}$ as a template for conductive silver material offers a green alternative for producing stable, cost-competitive and low-solid content conductive suspension. Optimization of the conductivity level can further be achieved by: (i) increase the silver loading in the suspension or (ii) eliminate the insulative cellulose after the synthesis. Preliminary trials were done by addition of sulfuric acid within the suspension to favor cellulose removal during the sintering step. However, up to now, acid addition leads to a loss of suspension stability and further works should then be done towards this way.
Table 5 Positioning of the developed ink towards conventional inkjet silver conductive ink properties

\begin{tabular}{lll}
\hline & Conventional ink ${ }^{47}$ & Our work \\
\hline Silver content $(\%)$ & $\approx 20 \%$ & $\approx 3 \%$ \\
$R_{\text {sh }}\left(\Omega \square^{-1}\right)$ & $0.1-0.5$ & $1-50$ \\
Minimal line width $(\mu \mathrm{m})$ & $50 \mu \mathrm{m}$ & $100 \mu \mathrm{m}$ \\
Sintering conditions & $110-200{ }^{\circ} \mathrm{C}$ & $180-250{ }^{\circ} \mathrm{C}$
\end{tabular}

\section{Conclusion}

The present work evaluates the possibility of using CNC to enhance both ink and substrate properties for printed electronics applications. The nanometrical size of CNC and their dispersing properties allow producing a compatible conductive silver inkjet ink. SEM images of the hybrid system evidence the presence of silver nanoparticles onto the CNC acting as a scaffold for the conductive material. A conductive ink at a low silver solid content was then achieved thanks to the CNC high aspect ratio and colloidal stability. Table 5 emphasizes the positioning of the developed ink towards inkjet silver conductive ink conventional properties.

Impact of CNC coating on the print quality was also investigated. This easy substrate pre-treatment leads to decrease the substrate roughness but also strongly increase the ink absorption kinetic of low volume. This faster absorption significantly limits the ink drop (picoliter) diffusion into a porous substrate, providing suitable properties for conductive tracks printing. The printing of the CNC-silver ink on such substrate allow producingconductive tracks with high resolution. However, it appears that the remaining $\mathrm{CNC}$ into the printed tracks limits the inks conductivity. Further works should then be focused on optimizing the obtained conductivity by either synthetizing conductive CNC prior the silver synthesis, eliminating the cellulose during or after the printing or trying others sintering strategies such as infrared or flash sintering on the printed film. Nevertheless, this innovative work provides a new solution to obtain conductive patterns on a porous substrate, with very low silver content within the dried film. The use of CNC as scaffold offers green way to produce a low-solid content conductive suspension. The produced conductive patterns on the CNC coating are then almost only composed of cellulosic material, thus offering a path towards renewable and sustainable electronics.

\section{Acknowledgements}

Authors acknowledge the French National Research Agency (ANRT) and Poly-Ink for the financial and material support of the PhD thesis. LGP2 is part of the LabEx Tec 21 (Investissements d'Avenir - grant agreement no. ANR-11-LABX-0030) and of the Energies du Futur and PolyNat Carnot Institutes. This research was made possible thanks to the facilities of the TekLiCell platform funded by the Région Rhône-Alpes (ERDF: European regional development fund). Authors also acknowledge Orlando Rojas for its advices towards a complementary study. 


\section{References}

1 A. Rida, R. Vyas and M. M. Tentzeris, IEEE Antenn. Propag. Mag., 2009, 51, 13-23.

2 M. Mäntysalo and P. Mansikkamäki, AEU - Int. J. Electron. Commun., 2009, 63, 31-35.

3 E. Sowade, K. Y. Mitra, E. Ramon, C. Martinez-Domingo, F. Villani, F. Loffredo, H. L. Gomes and R. R. Baumann, Org. Electron., 2016, 30, 237-246.

4 H. Yan, Z. Chen, Y. Zheng, C. Newman, J. R. Quinn, F. Dötz, M. Kastler and A. Facchetti, Nature, 2009, 457, 679-686.

5 W. Cao, J. Li, H. Chen and J. Xue, J. Photonics Energy, 2014, 4, 040990.

6 F.-G. Zeng, C.-C. Zhu, W. Liu and X. Liu, Microelectron. J., 2006, 37, 495-499.

7 T. Furukawa, N. Kawamura, J. Inoue, H. Nakada and M. Koden, Dig. Tech. Pap. - Soc. Inf. Disp. Int. Symp., 2015, 46, 1355-1358.

8 F. C. Krebs, M. Jørgensen, K. Norrman, O. Hagemann, J. Alstrup, T. D. Nielsen, J. Fyenbo, K. Larsen and J. Kristensen, Sol. Energy Mater. Sol. Cells, 2009, 93, 422-441.

9 D. Raghu and H. Peter, Printed, Organic \& Flexible Electronics Forecasts, Players \& Opportunities 2015-2025: IDTechEx, 2015.

10 B. Y. Tay and M. J. Edirisinghe, J. Mater. Sci., 2012, 37, 46534661.

11 F. Hoeng, A. Denneulin and J. Bras, Nanoscale, 2016, 8, 13131-13154.

12 J. Bras, D. Viet, C. Bruzzese and A. Dufresne, Carbohydr. Polym., 2011, 84, 211-215.

13 V. Kumar, R. Bollström, A. Yang, Q. Chen, G. Chen, P. Salminen, D. Bousfield and M. Toivakka, Cellulose, 2014, 21, 3443-3456.

14 K. Torvinen, J. Sievänen, T. Hjelt and E. Hellén, Cellulose, 2012, 19, 821-829.

15 Y. Fujisaki, H. Koga, Y. Nakajima, M. Nakata, H. Tsuji, T. Yamamoto, T. Kurita, M. Nogi and N. Shimidzu, Adv. Funct. Mater., 2014, 24, 1657-1663.

16 S. Ifuku, M. Tsuji, M. Morimoto, H. Saimoto and H. Yano, Biomacromolecules, 2009, 10, 2714-2717.

17 M. Wu, S. Kuga and Y. Huang, Langmuir, 2008, 24, 10494-10497. 18 W. G. Reed and J. O. Renn, US Pat., US2011026264, 2011.

19 O. Pras, D. Beneventi, D. Chaussy, P. Piette and S. TapinLingua, J. Mater. Sci., 2013, 48, 6911-6920.

20 Z. Shi, J. Tang, L. Chen, C. Yan, S. Tanvir, W. A. Anderson, R. M. Berry and K. C. Tam, J. Mater. Chem. B, 2014, 603-611.

21 N. Drogat, R. Granet, V. Sol, A. Memmi, N. Saad, C. Klein Koerkamp, P. Bressollier and P. Krausz, J. Nanopart. Res., 2010, 13, 1557-1562.

22 R. Xiong, C. Lu, W. Zhang, Z. Zhou and X. Zhang, Carbohydr. Polym., 2013, 95, 214-219.

23 G. Chinga-Carrasco, D. Tobjörk and R. Österbacka, J. Nanopart. Res., 2012, 14, 1213.

24 M.-C. Hsieh, C. Kim, M. Nogi and K. Suganuma, Nanoscale, 2013, 5, 9289-9295.

25 M. Nogi, N. Komoda, K. Otsuka and K. Suganuma, Nanoscale, 2013, 5, 4395-4399.
26 H. Koga, T. Saito, T. Kitaoka, M. Nogi, K. Suganuma and A. Isogai, Biomacromolecules, 2013, 14, 1160-1165.

27 F. Hoeng, A. Denneulin and J. Bras, Positive Impact of Cellulose Nanofibrils on Silver Nanowires Coatings for Transparent Conductive Films, Printed electronic conference, Santa Clara, 18-19 Nov 2015.

28 F. Navarro, M. Roman, A. R. Esker, R. M. Davis and S. H. Renneckar, Cellulose Nanocrystals: Size Characterization and Controlled Deposition by Inkjet Printing, ACS spring, San Francisco, 22-25 March 2010.

29 W. G. Reed and J. O. Renn, Electrically isolated heat sink for solid-state light, US20110026264 A1, 2011.

30 L. Zhang, E. G. Burns, T. Liu and J. A. Bates, Aqueous Dispersions Comprising Nanocrystalline Cellulose, and Compositions for Commercial Inkjet Printing, US2014267515, 2014.

31 Y. Habibi, H. Chanzy and M. R. Vignon, Cellulose, 2006, 13, 679-687.

32 F. Hoeng, A. Denneulin, C. Neuman and J. Bras, J. Nanopart. Res., 2015, 17, 244.

33 M. Binda, in Organic Electronics: principles, devices and applications, November 26-29th, 2013.

34 J. E. Fromm, IBM J. Res. Dev., 1984, 28, 322-333.

35 N. Reis and B. Derby, MRS Online Proc. Libr., 2011, 625, 117. 36 D. Jang, D. Kim and J. Moon, Langmuir, 2009, 25, 2629-2635.

37 R. D. Deegan, O. Bakajin, T. F. Dupont, G. Huber, S. R. Nagel and T. A. Witten, Nature, 1997, 389(6653), 827-829.

38 P. C. Duineveld, J. Fluid Mech., 2003, 477, 175-200.

39 D. Soltman and V. Subramanian, Langmuir, 2008, 24, 22242231.

40 H. Hu and R. G. Larson, J. Phys. Chem. B, 2006, 110, 70907094.

41 J.-L. Lin, Z.-K. Kao and Y.-C. Liao, Langmuir, 2013, 29, 1133011336.

42 E. Hrehorova, A. Pekarovicova, V. N. Bliznyuk and P. D. Fleming, Polymeric Materials for Printed Electronics and Their Interactions with Paper Substrates, in NIP \& Digital Fabrication Conference, Society for Imaging Science and Technology, 2007, pp. 928-931.

43 P. Antti, S. Jenni, T. Katariina, O. Kimmo and J. A. Ketoja, Cellulose, 2013, 1413-1424.

44 T. Lim, S. Han, J. Chung, J. T. Chung, S. Ko and C. P. Grigoropoulos, Int. J. Heat Mass Transfer, 2009, 52, 431-441.

45 A. Denneulin, J. Bras, A. Blayo, B. Khelifi, F. Roussel-Dherbey and C. Neuman, Nanotechnology, 2009, 20, 385701.

46 J. Ran, L. X. Mo, W. B. Li, W. W. Li, X. M. Fan, J. L. Fu and L. H. Li, Appl. Mech. Mater., 2012, 262, 501-504.

47 A. Kamyshny, J. Steinke and S. Magdassi, Open Appl. Phys. J., 2011, 19-36.

48 E. Tkalya, M. Ghislandi, W. Thielemans, P. van der Schoot, G. de With and C. Koning, ACS Macro Lett., 2013, 2, 157-163.

49 O. van den Berg, M. Schroeter, J. R. Capadona and C. Weder, J. Mater. Chem., 2007, 17, 2746. 